\title{
Prostate Artery Embolization
}

\author{
Samdeep Mouli, MD, MS ${ }^{1}$ Elias Hohlastos, MD ${ }^{1}$ Riad Salem, MD, MBA ${ }^{1}$
}

${ }^{1}$ Department of Radiology, Section of Interventional Radiology, Northwestern University, Chicago, Illinois

Semin Intervent Radiol 2019;36:142-148

Benign prostatic hyperplasia (BPH) involves the proliferation of the transition zone of the prostate, ${ }^{1,2}$ with resultant bladder outlet obstruction (BOO) and lower urinary tract symptoms (LUTS; frequency, urgency, weak stream, nocturia). The prevalence increases with age, affecting $50 \%$ of men in their 50s, with symptoms degrading quality of life (QoL). ${ }^{1,2}$ Prostatic arterial embolization (PAE) is safe and effective, demonstrating high rates of technical success, improved urinary flow rates, and QoL., 3

PAE can be a technically challenging and time-consuming procedure, especially given the general age of this patient population and concomitant medical comorbidities. Early enthusiasm in the procedure was tempered by variable clinical and technical success rates. ${ }^{5-9}$ These initial shortcomings were subsequently overcome by more standardization of techniques, ${ }^{10,11}$ and better understanding of patient selection and arterial anatomy. $3,7,12-14$

Utilizing this refined anatomical and procedural expertise, the goal of this article is to provide a standardized approach to PAE to overcome technical challenges described in the literature.

\section{Preprocedural Workup}

All patients considered for PAE are evaluated by a multidisciplinary team consisting of interventional radiologists and urologists. Inclusion and exclusion criteria have been described extensively elsewhere. ${ }^{3,15,16}$ A diagnosis of BOO secondary to BPH by urologic or urodynamic evaluation is critical to exclude other causes of LUTS not amenable to BPH treatment. ${ }^{17}$ All patients complete baseline LUTS questionnaires (International Prostate Symptom Score [IPSS], QoL, and International Index of Erectile Function [(IIEF]) to determine baseline degree of LUTS and erectile function. Baseline PSA is obtained and further evaluation with prostate biopsy is performed if clinically warranted. Further evaluation with crosssectional imaging (CT/MRI) may be obtained on a case-by-case basis depending on patient presentation and comorbidities.

Patients are started on ciprofloxacin prior to the procedure, which is continued for 5 days following the procedure.
Address for correspondence Samdeep Mouli, MD, MS, Department of Radiology, Northwestern University, 676 N. St. Clair, Suite 800, Chicago, IL 60611 (e-mail: s-mouli@northwestern.edu).

We do not routinely place a Foley catheter during the procedure, utilizing a condom catheter instead. An indwelling catheter is reserved for patients who intermittently catheterize themselves at least four to six times a day or have an indwelling catheter.

\section{Cone Beam CT}

The prostatic arteries typically arise from the anterior division of the internal iliac artery; however, the specific origin branch may vary, and may be asymmetric between sides of the gland. ${ }^{12-14,18}$ This variability has led to various angiographic classifcations $^{13,14,19}$ based on the branching pattern. Thus, knowledge of the vascular anatomy is critical to technical success. However, due to wide variability in both branching pattern and vascular anastomoses, performing several angiographic runs to delineate anatomy can be both time consuming and difficult, requiring multiple oblique images. Additionally, collateral vessels supplying adjacent pelvic organs often anastomose with the prostatic artery (PA). If these vessels are not clearly identified, there is an increased risk of nontarget embolization, and subsequent adverse events. ${ }^{13}$ Collateral extraprostatic supply is common and therefore should be interrogated prior to embolization of the PA. Several authors have demonstrated that coil embolization of these vessels is both safe and technically successful in preventing nontarget embolization. ${ }^{20,21}$

To prevent nontarget embolization, meticulous technique is necessary. In the case of PAE, this requires intraprocedural cone beam CT (CBCT) to confirm catheter placement and exclude nontarget embolization. ${ }^{18,22}$ In fact, CBCT provides essential information not seen during digital subtraction angiography (DSA) alone in $60.8 \%$ of cases. ${ }^{18}$ Additionally, a recent study demonstrated that $\mathrm{CBCT}$ identifies the PA with improved discrimination and less radiation dose than conventional preprocedural CTA. ${ }^{23}$

For these reasons, we perform $\mathrm{CBCT}$ at the start of all procedures ( - Table $\mathbf{1}$ ). Following arterial access, a 5 -Fr pigtail catheter is advanced into the abdominal aorta to the level of the iliac bifurcation. CBCT is then performed to map the origins of
Issue Theme Management of Complications in IR; Guest Editor, Robert K. Ryu, MD, FSIR

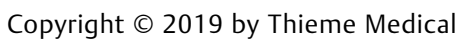
Publishers, Inc., 333 Seventh Avenue, New York, NY 10001, USA. Tel: +1(212) 584-4662.
DOI https://doi.org/ 10.1055/s-0039-1688431. ISSN 0739-9529. 
Table 1 Steps in the preprocedural evaluation and embolization procedure

\begin{tabular}{|l|l|}
\hline Step & Description \\
\hline Pelvic aortogram CBCT & $\begin{array}{l}\text { Settings: Artis Q (Siemens Medical Solutions) } \\
\text { with DynaCT (6 s acquisition) } \\
5 \mathrm{~mL} / \mathrm{s} \text { for } 12 \mathrm{~s} \text { at } 800 \mathrm{PSI}\end{array}$ \\
\hline $\begin{array}{l}\text { Right internal iliac artery } \\
\text { catheterization and angiography }\end{array}$ & DSA $3 \mathrm{~mL} / \mathrm{s} ; 12 \mathrm{~mL}$ volume \\
\hline Right PA catheterization, angiography \pm CBCT & RAO/CC oblique determined from initial pelvic CBCT \\
\hline Embolize right PA & Exclude nontarget embolization \\
\hline $\begin{array}{l}\text { Left internal iliac artery } \\
\text { catheterization and angiography }\end{array}$ & $\begin{array}{l}\text { Standard anatomy: Sos catheter selection using roadmap } \\
\text { Tortuous anatomy: 7-Fr sheath support catheter technique } \\
\text { DSA 3 mL/s; } 12 \text { mL volume }\end{array}$ \\
\hline Left PA catheterization, angiography \pm CBCT & LAO/CC oblique determined from initial pelvic CBCT \\
\hline Embolize left PA & Exclude nontarget embolization \\
\hline
\end{tabular}

Abbreviations: CBCT, cone beam computed tomography; CC, craniocaudal; DSA, digital subtraction angiography; LAO, left anterior oblique; PA, prostatic artery; RAO, right anterior oblique.

both prostatic arteries. Pelvic CBCT is useful for several reasons. The origin of the PA can be identified bilaterally, as well as the optimal oblique for visualization during ipsilateral iliac angiography. We have found that while an ipsilateral anterior oblique projection with cranial angulation is needed to identify the PA origin, given the oftentimes concomitant vessels tortuosity, the typical 35-degree ipsilateral anterior oblique/ 10-degree cranial angulation is not sufficient, often due to the overlapping appearance of pelvic vessels. ${ }^{23}$ Rather than perform several angiography runs to determine optimal positioning and separate overlapping vessels, a single pelvic CBCT allows us to visualize the number of prostatic arteries, their origins, and the angulation necessary to catheterize. This information also helps catheter and guidewire selection. Pelvic CBCT also improves prostate artery identification when used in conjunction with DSA. ${ }^{18}$ Additionally, CBCT can identify anastomoses with the vesical, rectal, and internal pudendal arteries such that coil embolization of these vessels can be performed prior to PA catheterization. ${ }^{20}$ Finally, several of the previously identified pitfalls and artifacts of $\mathrm{CBCT}^{24}$ can be mitigated with this overview of pelvic arterial anatomy, which may make it feasible to forgo subsequent interrogation of nontarget vessels. CBCT in conjunction with DSA delineates prostatic vascular supply, the flow characteristics of the prostatic vascular bed, which vessels require treatment, and the likelihood of nontarget embolization. By implementing this CBCT approach, we observed no cases of bladder, rectal, or penile ischemia. $^{22}$

\section{Vessel Catheterization}

Atherosclerosis commonly affects the iliac vasculature, and can lead to technical difficulties during PAE. ${ }^{25}$ Given the concomitant comorbidities in this patient population, tortuous common or internal iliac arteries are often encountered. Additionally, the prostatic arteries often arise at acute angles, and exhibit atherosclerotic narrowing. Furthermore, the degree of atherosclerotic narrowing is significantly correlated with prostate size and BPH symptoms, ${ }^{26,27}$ such that the most symptomatic patients may have the most challenging anatomy. These factors have been associated with increased technical difficulty when performing PAE. ${ }^{28}$ Given these constraints, using conventional microcatheter/microwires in the anterior division may not provide sufficient directionality or support to enable vessel catheterization. In these cases, we have used a modified approach adapting techniques used for the treatment of peripheral arterial disease.

In the setting of severe iliac tortuosity or atherosclerosis, we typically proceed with treatment of the side ipsilateral to our initial arterial access. A 7-Fr vascular sheath is placed with a 035 Amplatz wire advanced into the abdominal aorta to straighten out any vascular tortuosity. In a side-by-side fashion through the sheath, the ipsilateral internal iliac artery is selected with the operator's reverse curve catheter of choice (Sos, Simmons 1 or 2). The buddy-wire in the abdominal aorta facilitates both catheter stability and maneuverability. If support from the base catheter is insufficient, we exchange for a 5-Fr Kumpe catheter. This is advanced into the anterior division as distal as possible. This provides increased proximal support and improved directionality for microcatheter PA selection (- Fig. 1). Based on the initial pelvic $\mathrm{CBCT}$, the image intensifier is adjusted ipsilateral oblique/cranial angulation needed for vessel catheterization (-Fig. 2). A DSA is then performed from this angulation to visualize the PA origin. During DSA, opacification of the entire anterior division is necessary to guarantee visualization of both prostatic supply and any potential nontarget vessels, including any distal contribution from the internal pudendal or obturator arteries. ${ }^{29}$ As discussed extensively in prior anatomic reports, once the PA origin is identified, catheterization is performed using the road-map technique, using a $\leq 2.4=$ Fr microcatheter (Merit, Salt Lake City, ID; Terumo, Somerset, NJ), with either a 0.014 " or $0.016^{\prime \prime}$ shapeable hydrophilic microwires (Boston Scientific, Natick, MA; Stryker, Fremont, CA). Once the PA is catheterized, a suitable portion for embolization must be selected. Primarily, this must be beyond any potential nontarget vessels. Additionally, this must be proximal to any potential intraprostatic branch points to ensure thorough embolization of the gland. Care must be taken when addressing intraprostatic 


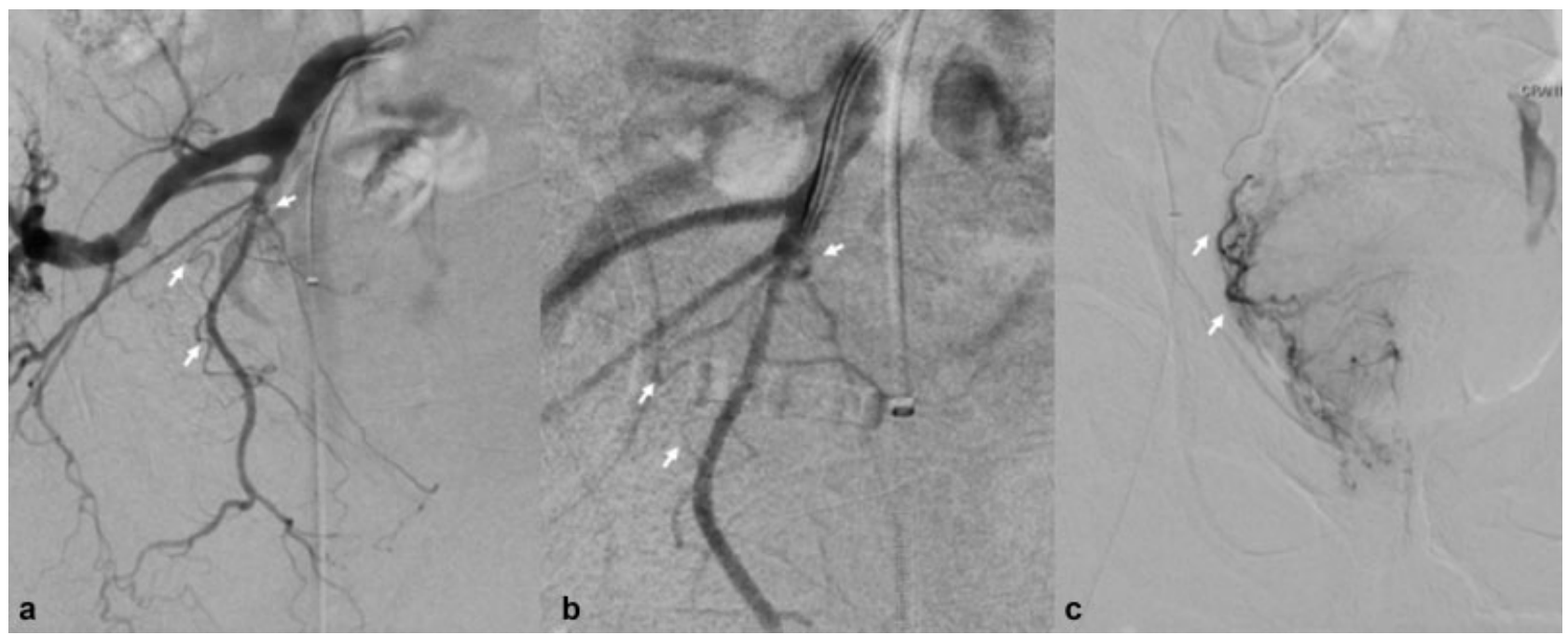

Fig. 1 (a) Right internal iliac angiography through Sos catheter demonstrating acute, tortuous origin of the prostatic artery (arrows) from proximal internal pudendal artery. (b) Base catheter exchanged for Kumpe catheter which is advanced deep into the anterior division of internal iliac artery, proximal to prostatic artery origin (arrows). (c) Microcatheter advanced through base catheter into prostatic artery (arrows).

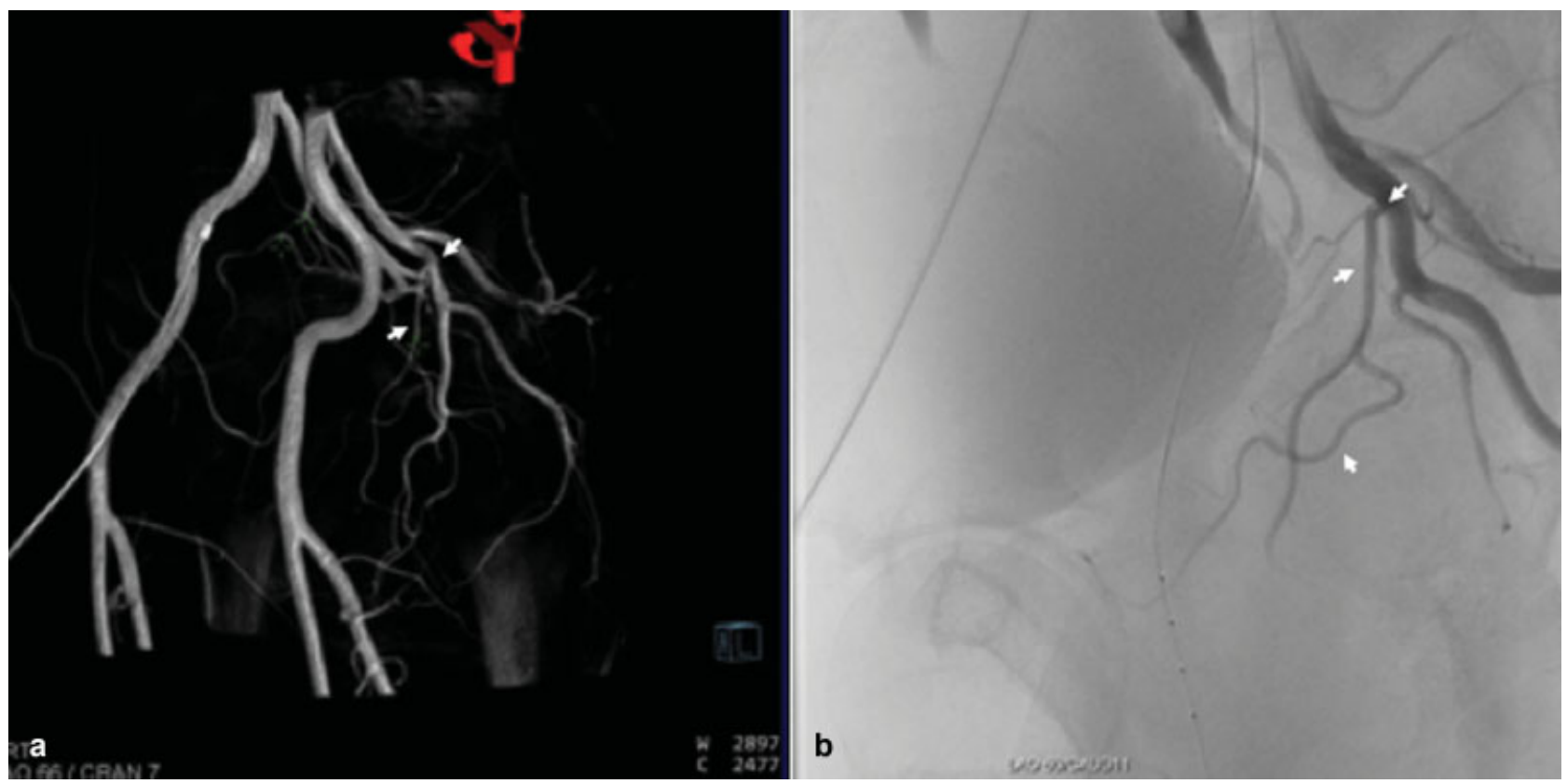

Fig. 2 (a) Pelvic CBCT volume rendered image demonstrating left anterior oblique angulation needed to visualize origin of left prostatic artery (white arrows) from anterior division of left internal iliac artery. (b) Left internal iliac artery DSA in same oblique determined by prior CBCT demonstrating origin of left prostatic artery (white arrows).

vascular branching, specifically the anterior/lateral prostatic artery and the posterior/lateral prostatic artery. The former is the principal focus of PAE, supplying the central gland tissue. The latter generally provides capsular supply, and may have anastomoses to extraprostatic tissues, such as the rectum or penis.

Special attention must be given to the case where the PA arises from a vesicoprostatic trunk from the proximal internal pudendal artery (-Fig. 3 ). The acute angulation of the PA origin here has been demonstrated to make catheterization difficult. ${ }^{28}$ In these cases, we utilize a tight reverse curve catheter, either a TCBNK or modified Sos catheter (Cook, Bloomington, IN), advanced beyond the vessel origin. From this location, the catheter is slowly retracted until it engages the vesico-prostatic trunk. From this location, a microcatheter and wire are advanced distally. Alternatively, this maneuver can be performed using an angled microcatheter or tip deflecting microcatheter forming a tight J-shape (-Fig. 4).

For the contralateral side, in routine cases without significant tortuosity, we advance a Sos catheter over the aortic bifurcation, using a glidewire advanced into the contralateral internal iliac artery. In cases of vessel tortuosity, we have modified catheterization techniques used for the treatment of peripheral arterial disease, termed the "reinforced sheath technique." A 5-Fr Sos catheter (Cook) is advanced over the guidewire to just above the iliac bifurcation. If needed, an 


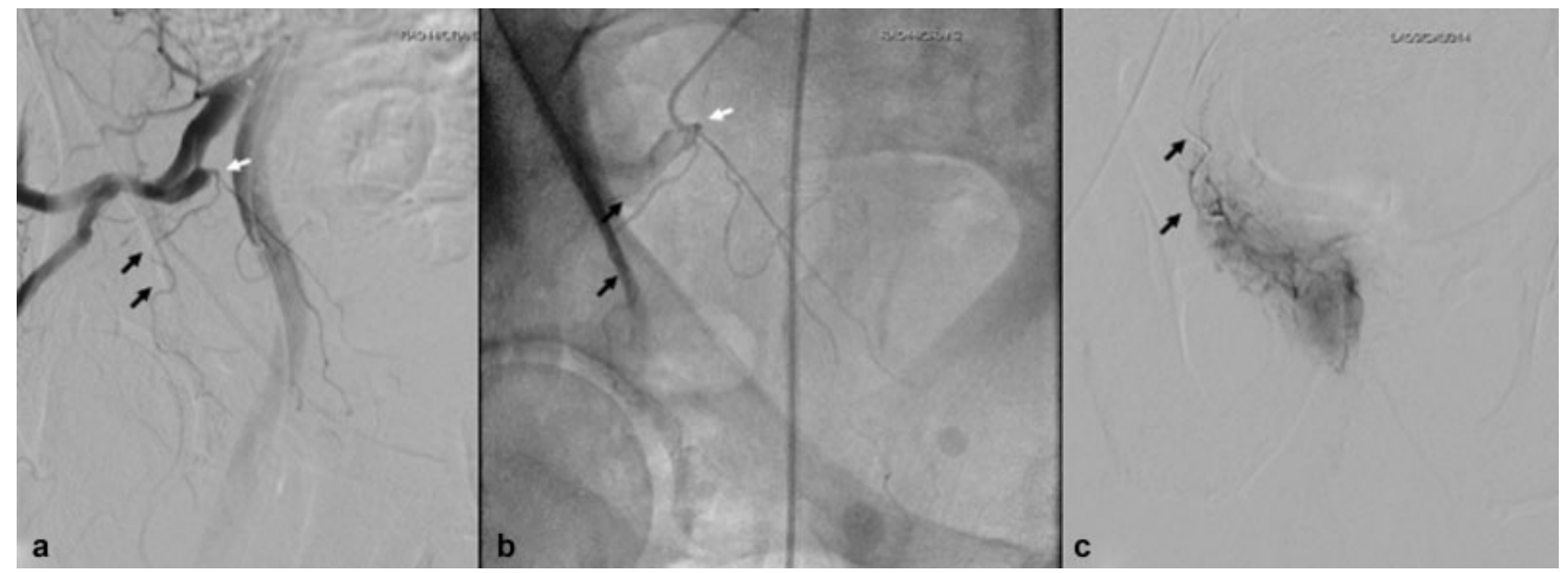

Fig. 3 (a) Right internal iliac angiography demonstrating proximal stenosis of the anterior internal iliac artery as well as vesicoprostatic trunk (white arrow), and subsequent prostatic artery (black arrows). (b) TCBNK catheter used to engage vesicoprostatic trunk, with DSA demonstrating opacification of prostatic artery (white arrow). (c) Microcatheter advanced through base catheter into prostatic artery with DSA demonstrating prostatic perfusion (black arrows).
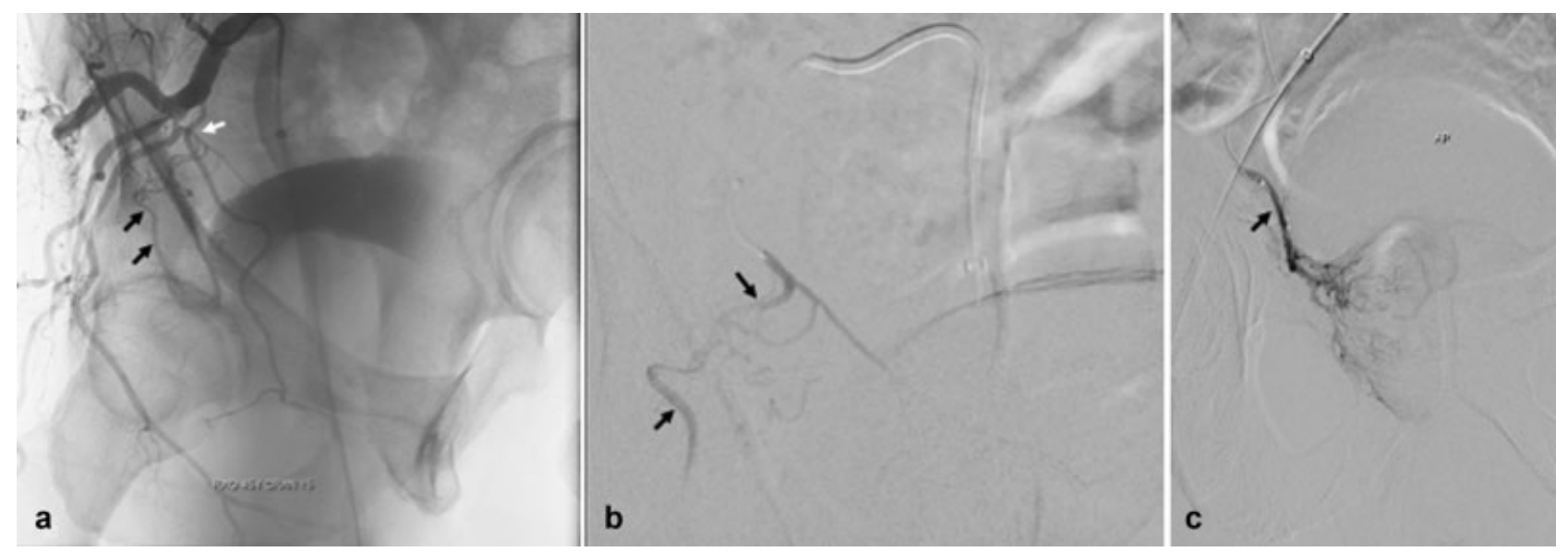

Fig. 4 (a) Right internal iliac angiography demonstrating atherosclerotic plaque in proximal anterior division of internal iliac artery and vesicoprostatic trunk (white arrow), and subsequent prostatic artery (black arrows). (b) Swift ninja microcatheter catheter used to engage vesicoprostatic trunk, with DSA demonstrating opacification of prostatic artery (black arrows). (c) Microcatheter advanced through base catheter into prostatic artery (black arrow) with DSA demonstrating prostatic perfusion.

arteriogram is performed to assess the iliac arteries in the contralateral oblique. Using the arteriogram as a guide with roadmap, a 0.035-in stiff angled glidewire (Terumo) is advanced through the SOS Omni catheter up and over the aortic bifurcation, and the catheter is advanced over the glidewire into the contralateral external iliac artery. The 5-Fr catheter and sheath are removed over the wire and exchanged for a 7-Fr, $45-\mathrm{cm}$ sheath (Terumo), which is advanced over the aortic bifurcation into the contralateral external iliac artery. Next, the sheath dilator is removed, and as the sheath is pulled back over the wire, contrast is injected through the sheath to identify the internal iliac artery origin. Once identified, the internal iliac and subsequently the anterior division can be catheterized with a combination of a 5-Fr Kumpe catheter and glidewire in a side-by-side fashion. The stiff glidewire (safety wire) and reinforced sheath provides stable access during these maneuvers and aids in subsequent catheter maneuverability. From this point, the Kumpe catheter can be directed toward the PA origin in the ipsilateral oblique/cranial angulation determined by the initial CBCT (-Fig. 5). DSA is performed to identify the PA origin, as discussed earlier.

\section{Embolization}

Once catheterized, angiography is performed in the ipsilateral oblique and frontal projections to demonstrate adequate filling of the prostatic vascular bed, as well as potential nontarget vessels. These may be seen in 10 to $20 \%$ of patients. ${ }^{30,31}$ However, a distinction must be made between small and large extraprostatic shunts. The former is frequently not visible on DSA unless a high-powered injection is employed. In the context of slow-flow embolization utilized in PAE, nontarget delivery of particles to these vessels is not encountered due to preferential flow to the prostate. ${ }^{20,30}$ In the case of larger shunts that are visible on DSA or low-pressure injection, these may either be avoided through advancement of the microcatheter distal to their takeoff or with coil embolization. The 


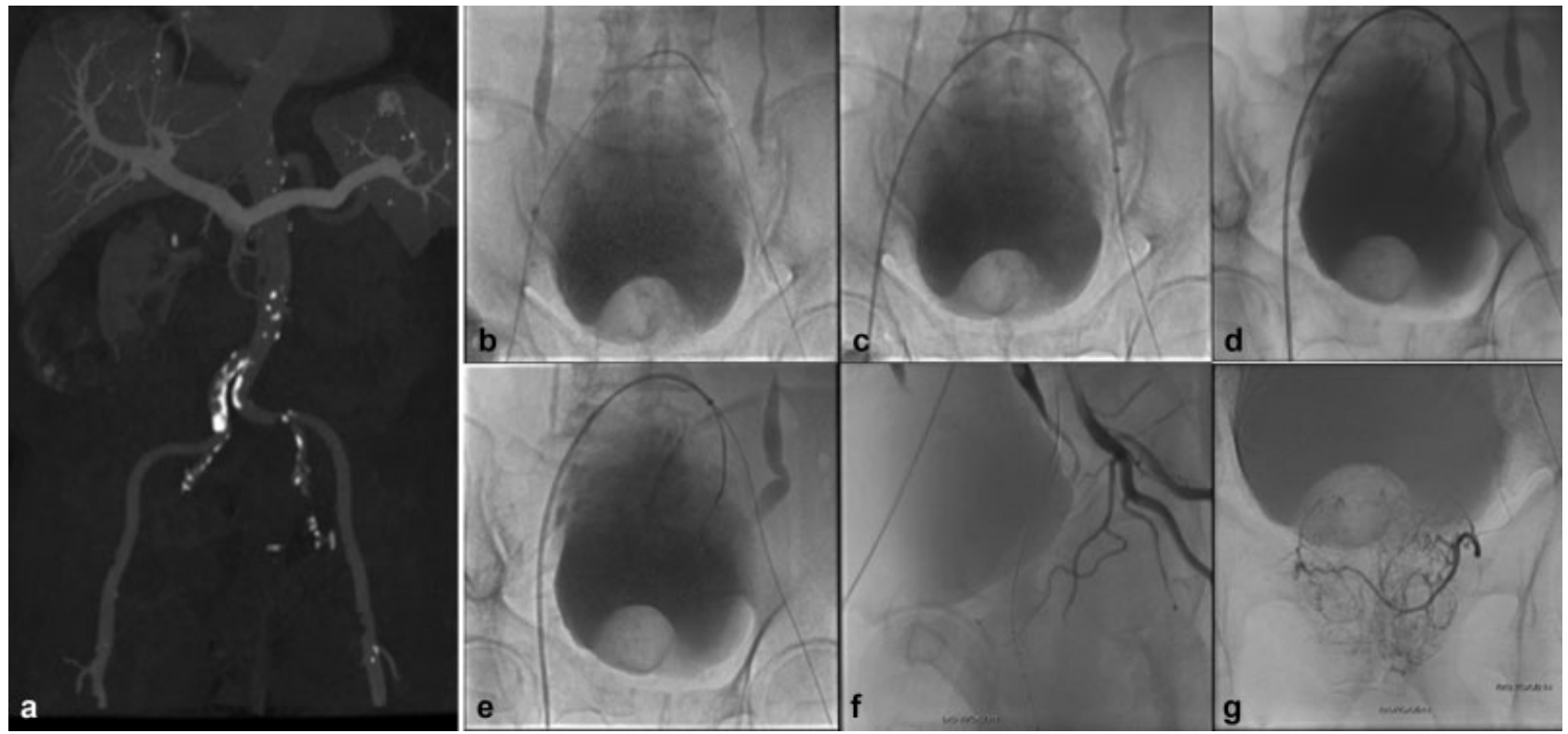

Fig. 5 (a) Coronal MIP of aortic bifurcation demonstrating high aortoiliac bifurcation and severe tortuosity. (b) AP view of the pelvis with Sos catheter at the level of the aortic bifurcation, with glidewire advanced into contralateral external iliac artery. (c) 7-Fr sheath advanced over the bifurcation into contralateral external iliac artery over sheath dilator. (d) Sheath withdrawn over the glidewire with injection of contrast demonstrating origin of internal iliac artery. (e) Kumpe catheter and second glidewire advanced into internal iliac artery, with original glidewire used as buddy-wire for support. (f) Left internal iliac angiography through Kumpe catheter demonstrating origin of prostatic artery. $(\mathbf{g})$ Microcatheter advanced into prostatic artery demonstrating prostatic perfusion.

vessels at risk of nontarget embolization have been described extensively elsewhere, ${ }^{30}$ and include superior vesicle, rectal, and pudendal branches. These anastomoses can be safely coil embolized without compromising therapeutic efficacy or increasing adverse events. ${ }^{20}$ The authors favor the use of small 3-mm pushable coils in these cases (-Fig. 6). Early in one's PAE experience, it may be prudent to coil embolize all potential nontarget vessels within the treatment zone, to avoid off-target microsphere deposition. With increased technical expertise, coil embolization of these vessels may not be necessary as long as injection pressure during DSA/embolization does not exceed inflow from these extraprostatic vessels, such that this inflow can be considered protective.

We perform embolization with 300 to $500 \mu \mathrm{m}$ particles, with several studies demonstrating equivalent efficacy and decreased adverse events compared with 100 to $300 \mu \mathrm{m}$ particles and nonspherical PVA. ${ }^{10,11}$ Unlike other vascular beds, embolization during PAE is a pressure-dependent, rather than flow-directed, phenomenon, due to small arterial caliber. As such, increased injection pressure can increase the likelihood of reflux and nontarget embolization. We therefore perform a slow controlled embolization using high dilution of particles to contrast. ${ }^{7,32,33}$ With the use of the previously described techniques with regard to artery identification, intraprocedural CBCT, and coil embolization, we have observed no cases of bladder, rectal, or penile ischemia, and a favorable adverse event profile. ${ }^{15}$

Steps in the preprocedural evaluation and embolization procedure for prostate embolization are outlined in - Table 1.

\section{Post-procedural Management}

Following recovery, patients are asked to void. If their postvoid residual volume (PVR) is less than $200 \mathrm{~mL}$ or in line with their screening PVR, they are subsequently discharged. Otherwise the voiding trial and PVR are repeated. Patients with chronic indwelling Foley catheters are instructed to return in 2 to 3 weeks for a voiding trial. Several series have reported successful voiding trials in the majority of these patients 10 to 20 days post-PAE, which has been concordant with our own experience. ${ }^{3,6,15,34}$

Patients are discharged with nonsteroidal anti-inflammatories, a proton pump inhibitor, and antibiotics for 5 days for potential post-PAE syndrome. This typically consists of urinary frequency and dysuria lasting up to 5 days. ${ }^{22}$ Patients are subsequently seen $1,3,6$, and 12 months following PAE for clinical assessment. Imaging assessment with postprocedural MRI is obtained at 6 months if comparative preprocedural imaging is available. From our own series, we have observed a 11- to 12-point reduction in IPSS and a 2- to 3-point improvement in QoL up to 1 year following PAE, similar to other series. ${ }^{15}$ We observed no severe adverse events, nontarget embolization, or adverse effects on erectile function or sexual health. ${ }^{15}$ On follow-up MR imaging, we saw a $26 \%$ decrease in central gland volume, including a $25 \%$ reduction in intravesical prostatic protrusion or median lobe size, again in line with other imaging series. ${ }^{35-37}$

PAE is both a safe and effective treatment for $\mathrm{BPH}$; however, meticulous technique is required for both technical and clinical success. Anatomic variability and tortuous atherosclerotic vasculature can pose significant technical challenges. Additionally, one must be wary of extraprostatic anastomoses that can increase the risk of nontarget embolization. A methodical approach to angiography, incorporating techniques utilized in the treatment of peripheral vascular disease, can facilitate optimal outcomes. 


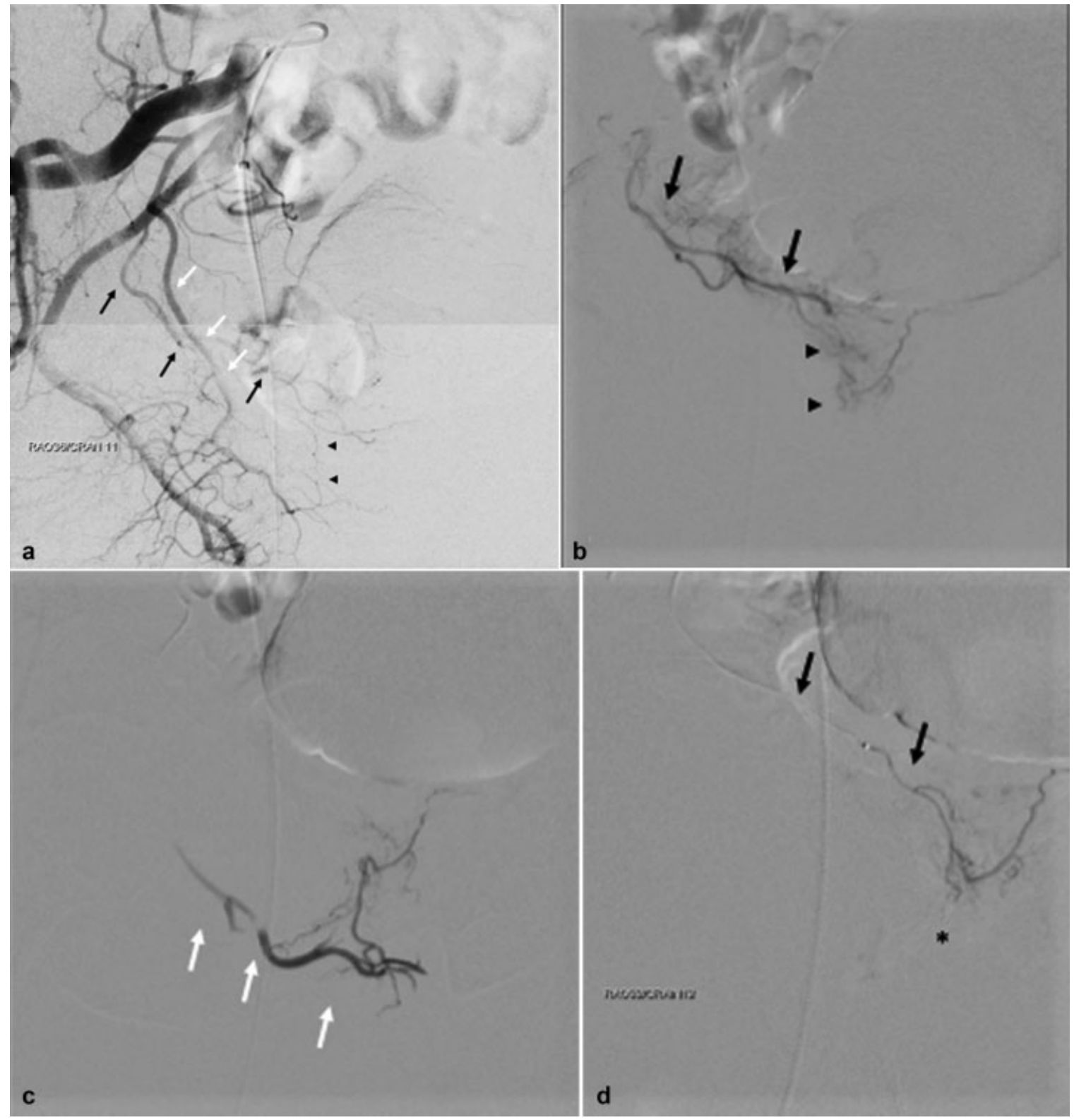

Fig. 6 (a) Right internal iliac angiography demonstrating origin of the prostatic artery (black arrows), as well as obturator artery (white arrows). Prostatic perfusion demonstrating potential intraprostatic anastomosis between prostatic and obturator arteries (arrow heads). (b) Right prostatic artery (black arrows) DSA demonstrating arterial anastomosis (arrow heads) extending beyond prostatic borders. (c) Microcatheter advanced through anastomosis with DSA demonstrating reflux into obturator artery (white arrows). (d) Microcatheter retracted into main prostatic artery following coil embolization of the anastomosis (asterisk). Prostatic perfusion seen without opacification of obturator anastomosis (arrows).

\section{Funding}

This study was funded by Northwestern Medicine.

\section{Conflict of Interest}

None.

\section{References}

1 McVary KT, Roehrborn CG, Avins AL, et al. Update on AUA guideline on the management of benign prostatic hyperplasia. J Urol 2011;185(05):1793-1803. doi: 10.1016/j.juro.2011.01.074

2 Roehrborn CG. Pathology of benign prostatic hyperplasia. Int J Impot Res 2008;20(Suppl 3):S11-S18
3 Pisco JM, Bilhim T, Pinheiro LC, et al. Medium- and long-term outcome of prostate artery embolization for patients with benign prostatic hyperplasia: results in 630 patients. J Vasc Interv Radiol 2016;27(08):1115-1122

4 Kurbatov D, Russo GI, Lepetukhin A, et al. Prostatic artery embolization for prostate volume greater than $80 \mathrm{~cm} 3$ : results from a single-center prospective study. Urology 2014;84(02):400-404

5 Kaplan SA. Prostate arterial embolization is a viable option for treating symptoms of benign prostatic hyperplasia: con. J Urol 2017;198(01):9-11

6 Carnevale FC, da Motta-Leal-Filho JM, Antunes AA, et al. Quality of life and clinical symptom improvement support prostatic artery embolization for patients with acute urinary retention caused by benign prostatic hyperplasia. J Vasc Interv Radiol 2013;24(04): 535-542 
7 Pisco J, Campos Pinheiro L, Bilhim T, et al. Prostatic arterial embolization for benign prostatic hyperplasia: short- and intermediate-term results. Radiology 2013;266(02):668-677

8 Bagla S, Martin CP, van Breda A, et al. Early results from a United States trial of prostatic artery embolization in the treatment of benign prostatic hyperplasia.J Vasc Interv Radiol 2014;25(01):47-52

9 Gao YA, Huang Y, Zhang R, et al. Benign prostatic hyperplasia: prostatic arterial embolization versus transurethral resection of the prostate-a prospective, randomized, and controlled clinical trial. Radiology 2014;270(03):920-928

10 Gonçalves OM, Carnevale FC, Moreira AM, Antunes AA, Rodrigues VC, Srougi M. Comparative study using $100-300$ versus $300-500$ $\mu \mathrm{m}$ microspheres for symptomatic patients due to enlarged-BPH prostates. Cardiovasc Intervent Radiol 2016;39(10):1372-1378

11 Hwang JH, Park SW, Chang IS, et al. Comparison of nonspherical polyvinyl alcohol particles and microspheres for prostatic arterial embolization in patients with benign prostatic hyperplasia. BioMed Res Int 2017;2017:8732351

12 Bilhim T, Tinto HR, Fernandes L, Martins Pisco J. Radiological anatomy of prostatic arteries. Tech Vasc Interv Radiol 2012;15 (04):276-285

13 Bhatia S, Sinha VK, Abdul-Rahim O, Harward S, Narayanan G. Rare prostatic artery origins and the importance of collateral circulation in prostate artery embolization: a pictorial essay. Can Assoc Radiol J 2018;69(02):220-229

14 Amouyal G, Pellerin O, Del Giudice C, Dean C, Thiounn N, Sapoval M. Variants of patterns of intra- and extra-prostatic arterial distribution of the prostatic artery applied to prostatic artery embolization: proposal of a classification. Cardiovasc Intervent Radiol 2018;41(11):1664-1673

15 Salem R, Hairston J, Hohlastos E, et al. Prostate artery embolization for lower urinary tract symptoms secondary to benign prostatic hyperplasia: results from a prospective FDA-approved investigational device exemption study. Urology 2018;120:205-210

16 Isaacson AJ, Raynor MC, Yu H, Burke CT. Prostatic artery embolization using Embosphere Microspheres for prostates measuring $80-150 \mathrm{~cm}$ (3): early results from a US trial. J Vasc Interv Radiol 2016;27(05):709-714

17 Gomez C, Bhatia S, Carnevale FC, Narayanan G. Role of urodynamic studies in management of benign prostatic obstruction: a guide for interventional radiologists. J Vasc Interv Radiol 2017;28(01): 126-133

18 Wang MQ Duan F, Yuan K, Zhang GD, Yan J, Wang Y. Benign prostatic hyperplasia: cone-beam CT in conjunction with DSA for identifying prostatic arterial anatomy. Radiology 2017;282(01): 271-280

19 de Assis AM, Moreira AM, de Paula Rodrigues VC, et al. Pelvic arterial anatomy relevant to prostatic artery embolisation and proposal for angiographic classification. Cardiovasc Intervent Radiol 2015;38(04):855-861

20 Bhatia S, Sinha V, Bordegaray M, Kably I, Harward S, Narayanan G. Role of coil embolization during prostatic artery embolization: incidence, indications, and safety profile. J Vasc Interv Radiol 2017;28(05):656-664.e3

21 Isaacson AJ, Bhalakia N, Burke CT. Coil embolization to redirect embolic flow during prostatic artery embolization. J Vasc Interv Radiol 2015;26(05):768-770

22 Moreira AM, de Assis AM, Carnevale FC, Antunes AA, Srougi M, Cerri GG. A review of adverse events related to prostatic artery embolization for treatment of bladder outlet obstruction due to BPH. Cardiovasc Intervent Radiol 2017;40(10):1490-1500

23 Desai H, Yu H, Ohana E, Gunnell ET, Kim J, Isaacson A. Comparative analysis of cone-beam CT angiogram and conventional CT angiogram for prostatic artery identification prior to embolization.J Vasc Interv Radiol 2018;29(02):229-232

24 Bagla S, Sterling KM. Pitfalls of cone beam computed tomography in prostate artery embolization. Cardiovasc Intervent Radiol 2014;37(06):1430-1435

25 Mohan PP, Sandhu J, Kably I. Retrograde prostatic arterial embolization via penile collateral in chronic total occlusion of the prostatic artery. J Vasc Interv Radiol 2018;29(09):1331-1333

26 Haga N, Akaihata H, Hata J, et al. The association between local atherosclerosis of the prostatic artery and benign prostatic enlargement in humans: Putative mechanism of chronic ischemia for prostatic enlargement. Prostate 2018;78(13):1001-1012

27 Cimadamore A, Scarpelli M, Santoni M, et al. Re: Friederike Haidl, David Pfister, Axel Heidenreich. Re: Prostatic artery embolization in the treatment of localized prostate cancer: a bicentric prospective proof-of-concept study of 12 patients. Mordasini L, Hechelhammer L, Diener PA, et al. J Vasc Interv Radiol 2018;29:589-97. Eur Urol 2018;74:525-6: Could morphologic changes in periprostatic arteries have an influence on prostatic artery embolization? Eur Urol 2019;75(04):e110-e113

28 du Pisanie J, Abumoussa A, Donovan K, Stewart J, Bagla S, Isaacson A. Predictors of prostatic artery embolization technical outcomes: patient and procedural factors. J Vasc Interv Radiol 2019;30(02): 233-240

29 Isaacson AJ, Piechowiak RL, Nutting C, Bagla S. How to "get out of trouble" during prostatic artery embolization. Tech Vasc Interv Radiol 2018;21(04):288-294

30 Amouyal G, Chague P, Pellerin O, et al. Safety and efficacy of occlusion of large extra-prostatic anastomoses during prostatic artery embolization for symptomatic BPH. Cardiovasc Intervent Radiol 2016;39(09):1245-1255

31 Zhang G, Wang M, Duan F, et al. Radiological findings of prostatic arterial anatomy for prostatic arterial embolization: preliminary study in 55 Chinese patients with benign prostatic hyperplasia. PLoS One 2015;10(07):e0132678

32 Carnevale FC, Antunes AA. Prostatic artery embolization for enlarged prostates due to benign prostatic hyperplasia. How I do it. Cardiovasc Intervent Radiol 2013;36(06):1452-1463

33 Bilhim T, Pisco JM, Rio Tinto H, et al. Prostatic arterial supply: anatomic and imaging findings relevant for selective arterial embolization. J Vasc Interv Radiol 2012;23(11):1403-1415

34 Bhatia S, Sinha VK, Kava BR, et al. Efficacy of prostatic artery embolization for catheter-dependent patients with large prostate sizes and high comorbidity scores. J Vasc Interv Radiol 2018;29 (01):78-84.e1

35 Ali R, Gabr A, Mouli SK, et al. MR imaging findings of the prostate gland following prostate artery embolization: results from a prospective phase 2 study. Abdom Radiol (NY) 2019;44(02): 713-722

36 Frenk NE, Baroni RH, Carnevale FC, et al. MRI findings after prostatic artery embolization for treatment of benign hyperplasia. AJR Am J Roentgenol 2014;203(04):813-821

37 Zhang H, Shen Y, Pan J, et al. MRI features after prostatic artery embolization for the treatment of medium- and large-volume benign hyperplasia. Radiol Med (Torino) 2018;123(10):727-734 